


\section{Rare and orphan lung diseases}

\section{Educational aims}

1 To motivate the interest of chest physicians in rare orphan lung diseases.

I To define rare and/or orphan lung diseases.

I To provide information on orphan drugs.

I To give examples of orphan lung diseases.

I To provide details about patient associations, networks and reference centres.

\section{Summary}

Patients with rare lung diseases often feel isolated, especially if their physician has limited experience and knowledge of their disease. They can feel like an "orphan" in the field of healthcare. A respiratory professional who does not work in a large hospital in a big town may not feel concerned by rare and so-called orphan lung diseases, since it is often supposed that these diseases are reserved for university specialists. However, this should not be the case, as patients live in different locations throughout every country and have proximity healthcare needs. Therefore, every respiratory professional should meet several patients with a rare disease in the course of their career. This review examines how to deal with such patients and where information can be obtained in order to offer the best current care.

\section{Key points}

I Patients suffering from rare and orphan lung diseases often feel frustrated and angry because of a delayed diagnosis and the insufficient experience of their nearest physician.

I As many diseases can be classed as rare or orphan, pulmonologists need to cooperate through networks and form connections with specialised reference centres to ensure optimal diagnosis and care.

I Participation in registries and clinical studies helps to increase the collective and individual knowledge of respiratory specialists.

I Providing information to patients both directly and through patients' associations is a necessity, as orphan diseases are not a regular subject covered in medical reports in the lay press.

I Action is being taken by the ERS to include rare and orphan lung diseases in scientific and educational programmes and to develop task forces for specific diseases.

\section{J-F. Cordier}

Professor of Respiratory Medicine - Claude Bernard University Head, Dept of Respiratory Medicine - Reference Centre for Orphan Pulmonary Diseases Louis Pradel University Hospital 69677 Lyon (Bron)

France

Fax: 33472357653

E-mail: jean-

francois.cordier@chu-lyon.fr 


\section{Rare and/or orphan?}

In Europe, a rare disease is defined as affecting less than one in every 2,000 people. However, this definition covers a marked range, since some disorders affect several thousands or tens of thousands of people, while others affect only a few hundred or dozens of patients. There are about 8,000 different rare diseases, the majority $(\sim 80 \%)$ of which are considered to be of genetic origin. Diseases of genetic origin may present at birth or during childhood, or may even only manifest at adulthood. Furthermore, the clinical phenotype of rare diseases may vary, further complicating diagnosis and care, especially when several organs are involved.
The term orphan disease means that almost nobody cares for patients with the disease and that no or little research has been undertaken to improve diagnosis, and to develop and commercialise drugs to treat the condition. Orphan diseases may be common diseases that are neglected in low-income countries, for example tropical diseases such as trypanosomiasis (i.e. African sleepiness disease and American Chagas disease) [1]. As people in such developing countries do not have the money to buy drugs, most pharmaceutical companies do not invest in research for these non-solvent populations. In most European countries, orphan diseases are rare diseases, which are little known to the public and even doctors, with minimal research interest

\section{Box 1 Mister Merrick and Doctor Treves: from curiosity to solicitude}

At the end of the 19th century, Joseph Merrick, so called the "Elephant Man", and Frederick Treves, a fashionable physician in London, offered the paradigm of the diagnosis and care of rare diseases, in particular the social and psychological distress of patients who are (or at least feel) orphan in the world of health.

From birth, Joseph Merrick was disfigured by multiple subcutaneous nodules, macrocephaly and widespread skin masses misshaping his body. He was very intelligent. The exact diagnosis of his condition is still debated (it was initially thought to be neurofibromatosis, but the diagnosis of

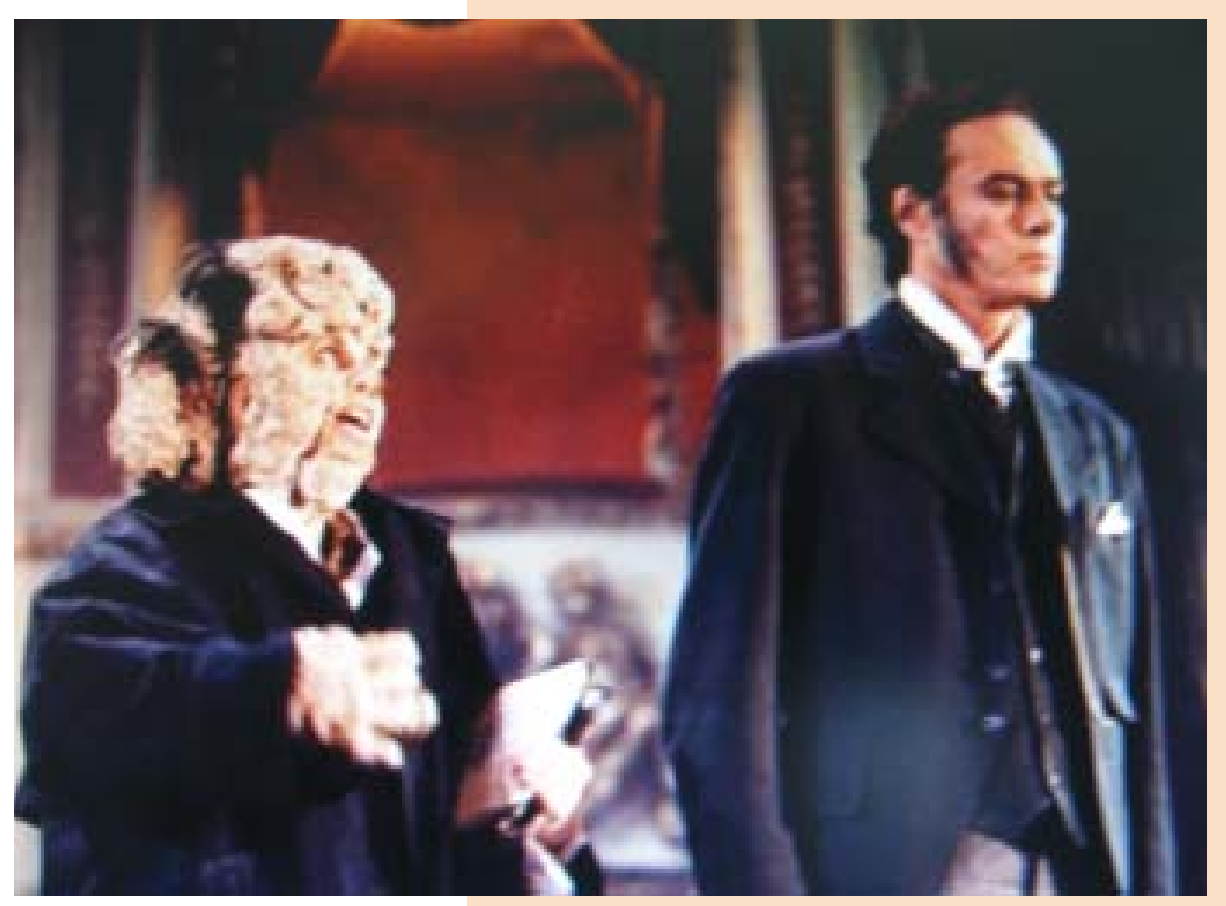
Proteus syndrome is now favoured).

The Elephant Man was exhibited in a circus of freaks in very poor and inhumane conditions. Treves heard of him, visited him, and began to develop an interest. However, this began as medical curiosity, with Treves exhibiting Merrick at the Pathological Society of London in 1884 (a presentation not very different from his circus display!). However, he progressively developed empathy for Merrick and admitted him to a London hospital, where Treves spent much time talking with him daily (and even every Sunday morning), thus allowing the patient to feel socially accepted. Famous people came and visited Merrick, who became very popular.

The fascinating story of the Elephant Man has been the subject of theatre plays, of a film by David Lynch (1980) and, more recently, of an opera by Laurent Petitgirard (figure 1; reproduced with permission from

www.petitgirard.com), who stated: "from Figure 1 a phenomenon we examine almost with voyeurism, Joseph Merrick becomes a mirror in which we discover our own fear of difference, so that in the end, when he questions the mercy of his God, we identify with him". 


\section{Box 2 When asthma becomes severe and atypical}

A 35-year-old non-smoking male patient who has suffered with asthma for 5 years presents to you. His asthma has been well controlled, until 6 months previous to his presentation, with inhaled long-acting $\beta_{2}$-agonists and corticosteroids. His asthma has progressively worsened over a period of 3 months, requiring oral courses of corticosteroids for 1 week or more to obtain almost complete improvement. The severity and frequency of relapses have increased dramatically, shortly after stopping oral corticosteroids, despite increased doses of inhaled fluticasone ( $500 \mu \mathrm{g}$ twice a day). On presentation, the patient complains about the high doses of corticosteroids that he is taking, and you face a dilemma: you were taught at university and then at a Postgraduate course to avoid prolonged oral corticosteroid treatment in asthma, but what other option do you have when the patient is doing so badly! The patient has stopped oral corticosteroids for 5 days and currently feels terrible. He has mild fever, weight loss ( $5 \mathrm{~kg}$ within the last 2 weeks), severe dyspnoea, pulmonary infiltrates on chest radiograph, palpable purpura of the legs, dysaesthesia and weakness in the left leg. His forced expiratory volume in one second is $1,200 \mathrm{~mL}(30 \%$ pred), without improvement with bronchodilators.

What should you do?

This patient has severe (difficult) asthma, which is steroid dependent, and he has also developed fixed airflow obstruction associated with fever and weight loss, as well as probable cutaneous vasculitis and mononeuritis. The rare Churg Strauss syndrome (CSS) must be suspected. The blood hallmark of CSS is eosinophilia. The white cell count that you ask for shows that the eosinophil count is $8 \times 10^{9}$ per L. A complete work-up is immediately necessary to define the extent of organ involvement (especially the heart) and to choose the appropriate treatment with the help of a specialist in the field.

for physicians and scientists. Therefore, patients feel like they are orphans in the healthcare world (box 1).

Patients often feel disappointed and angry, as their diagnosis may not be confirmed for months or even years until they eventually find a competent and experienced clinician. Diagnosis is especially difficult when the disease appears as a more common disorder (box 2).

Furthermore, teaching in medical schools cannot cover the thousands of previously identified rare diseases. As general practitioners can not know the signs and symptoms of all the 8,000 rare diseases, specialists must have the capacity to suspect different diagnoses in their own field, to be able to orientate the patient towards referral centres where the final diagnosis may be confirmed and management defined.

\section{Orphan lung diseases}

The orphan lung diseases or orphan diseases including lung involvement have not yet been indexed. Table 1 includes an indicative list of orphan lung diseases, which is far from exhaustive, but which illustrates examples of conditions varying in many aspects. Some of these are almost exclusively acquired respiratory diseases (e.g. idiopathic pulmonary fibrosis), others are orphan systemic diseases comprising pulmonary involvement that may represent the main mortality risk (e.g. cystic fibrosis, a disorder of genetic origin, scleroderma, an acquired disease), others affect only one sex (e.g. lymphangioleiomyomatosis (LAM), which only affects females) (box 3). Some diseases that were termed orphan someyears ago

\section{Table 1 Examples of orphan lung diseases}

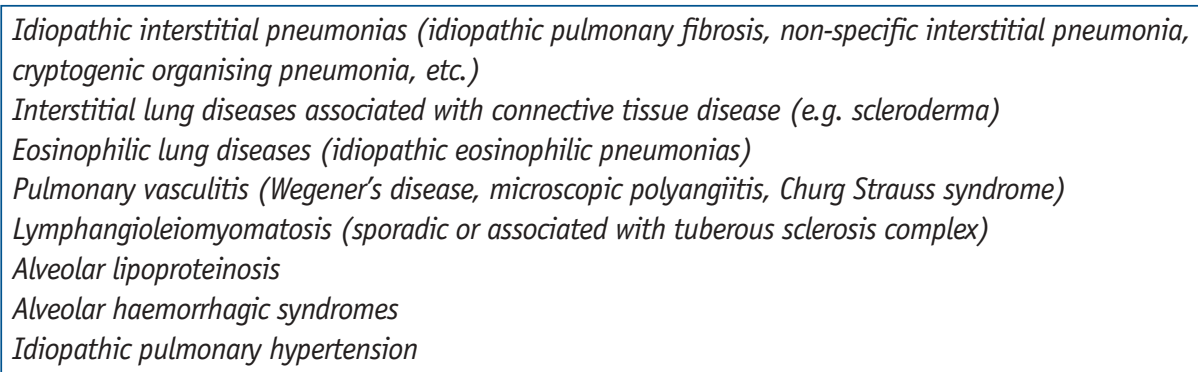




\section{Box 3 LAM: an example of orphan lung disease}

Pulmonary LAM is a disease affecting (almost) exclusively females of child-bearing age. It is due to the proliferation of abnormal smooth muscle cells involving peribronchovascular and lymphatic peripheral interstitium, thus resulting in destructive cystic lesions with a characteristic diffuse cystic pattern on HRCT and airflow obstruction. Recurrent pneumothoraces and increasing dyspnoea, and possible chylous effusions (chylothorax, chylous ascites) represent the main clinical manifestations. LAM may be sporadic, or associated with tuberous sclerosis complex (TSC), an autosomal dominant genetic disorder with systemic hamartomatosis. Both sporadic and TSC-associated LAM are linked with gene mutations of the genes TSC1 (encoding hamartin) and TSC2 (encoding tuberin) genes, which are tumour suppressor genes whose mutation induces cellular proliferation. No specific treatment of LAM is currently available. Lung transplantation is the only hope for patients with end-stage lung disease.

Figure 2 shows a chest HRCT of a patient with pulmonary LAM (associated with TSC), showing diffuse multiple thin-walled cysts distributed throughout the lungs. Figure 3 shows abdominal HRCT of the same patient showing bilateral angiomyolipomas, a characteristic finding in LAM either sporadic or associated with TSC.

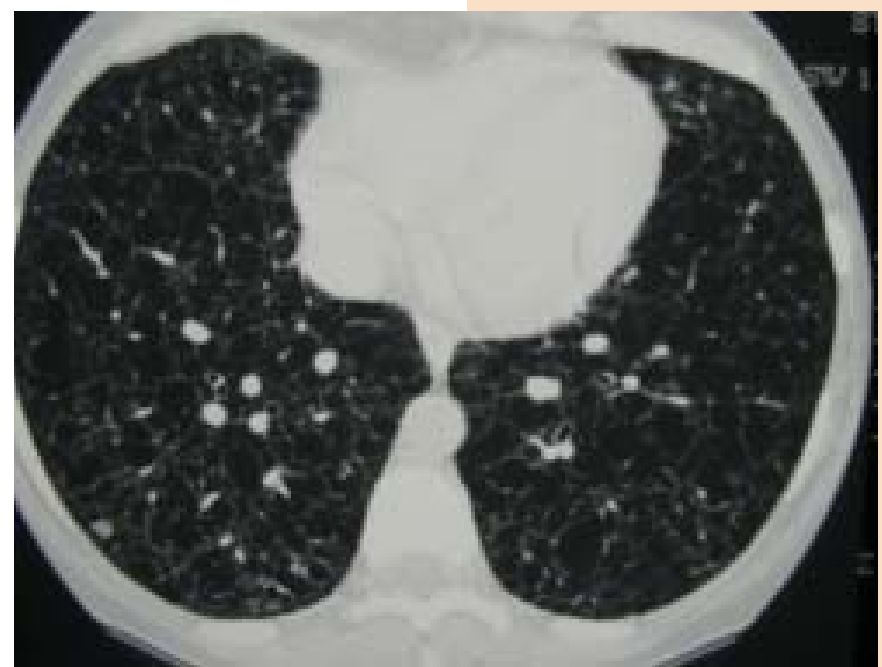

Figure 2

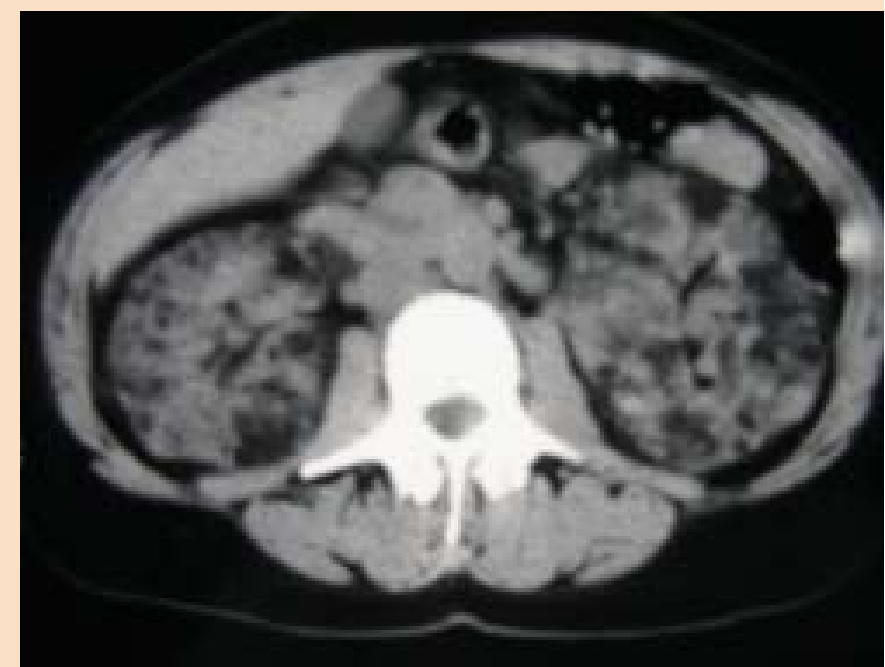

Figure 3 have now evolved into rare diseases, because a tremendous increase in research has both resulted from and resulted in efficient drug discovery and commercialisation (e.g. primary pulmonary hypertension).

\section{Learning about rare/orphan lung diseases}

The interest of chest physicians for these diseases has gradually increased over recent years for several reasons. New diagnostic tools (such as high-resolution computed tomography (HCRT)) have demonstrated more characteristic diagnostic patterns or results (e.g. the crazy-paving pattern of alveolar lipoproteinosis on chest HRCT or anti-neutrophil cytoplasmic autoantibodies with anti-proteinase 3 specificity at high titre in Wegener's granulomatosis). Furthermore, the demand of patients for adequate investigations and a definite diagnosis has markedly increased, specifically for those who obtain medical information from the Internet. It has become more common that a patient comes and says: "Doctor, I believe I have Wegener's disease", whether they have or haven't. It is, therefore, important that the chest physician knows where he can get correct, up-to-date information from in order to choose the appropriate investigations for the right diagnosis. Information can be obtained directly from original papers published in specialty journals (by searching on PubMed www.ncbi.nlm.nih.gov), but these are (too) often focused on basic research with limited immediate applicability. Journals and monographs (such as the European Respiratory Journal and the European Respiratory Monograph) provide some review articles where confident and comprehensive information may be found. Furthermore, websites are available for certain diseases or groups of diseases, such as 
Orphanet (www.orpha.net) and the National Organisation for Rare Disorders (NORD) (www.rarediseases.org).

The increasing interest in rare orphan lung diseases has further resulted in the organisation of Symposia and Postgraduate Courses at the annual ERS Congress. These are often overcrowded, proof of the need and interest of chest physicians for learning about rare diseases! Finally, personalised information may be obtained from most referral centres.

\section{Orphan drugs and other pathways for care}

The US Orphan Drug Act, brought into law in 1983 , has provided incentives to the pharmaceutical industry to improve rare disease drug development (i.e. market exclusivity, research tax credits, grants). However, financial success is rare. The American Cystic Fibrosis Foundation (Bethes$\mathrm{da}, \mathrm{MD}, \mathrm{USA}$ ) has poured large amounts of money into drug development, with only one return on clinical investment with aerosolised TOBI (tobramycin) [2]. In Europe, regulations proposed by the European Parliament and the Committee for Orphan Medical Products (COMP) were adopted in December 1999. The principle direct incentives comprise the designation procedure for orphan medicinal products (possibility of fee waivers, 10-year market exclusivity, protocol assistance, etc.). As of July 2005, 365 applications have been submitted to the European Medicines Evaluation Agency (EMEA) and COMP, and a total of 206 have been designated. However, a reduction of the product's market exclusivity to 6 years for drugs sufficiently profitable at 5 years is under discussion. The orphan drug regimen of the European Union (EU) may, thus, differ more from the US regimen, the efficiency of which has resulted in the approval of $\sim 50$ drugs, whereas the EU regimen has resulted in the approval of <20 [3]. Complementary national initiatives have further been taken by most European countries.

An example of an orphan drug in the pulmonary field is bosentan, approved for the treatment of idiopathic pulmonary hypertension. Much therapeutic research is currently pursued in this field.

Drugs designed to specifically treat one orphan disease remain rare, but other treatment pathways are multiple. Although gene therapy of cystic fibrosis still seems a far hope (evidence has been disappointing after the great expectations following the discovery of the cystic fibrosis transmembrane regulator (CFTR) gene mutations), continuous improvement has been obtained by research into various therapeutic pathways, including the control of infection and exacerbations, according to recommendations for patients, families and physicians in connection with the specialised cystic fibrosis care centres that have developed worldwide [4]. Many orphan lung diseases are also treated using common drugs, such as corticosteroids and immunosuppressors (e.g. pulmonary vasculitis). Therapeutic research has recently become active for idiopathic pulmonary fibrosis, after many years of therapeutic nihilism.

\section{Patients' associations}

For most rare diseases, associations of patients have emerged, usually under the dynamism of a patient or the parents of an affected child, and these play a specific role in helping patients with daily difficulties, distress and isolation. They help in translating the medical information about the disease and its care into common language, and often provide answers to the questions that many people do not dare to ask their doctor (e.g. urinary incontinence in females with severe cough due to interstitial lung disease). Patient associations, when powerful, play a useful role in the funding of medical research projects and the definition of guidelines. They must be independent from the medical specialists of the disease to play their role well, but their input is highly valuable in all the networks, referral centres and research projects. The size of the associations is extremely variable, largely dependent on the prevalence of the disease. For example, associations of patients with cystic fibrosis are extremely powerful throughout the world. Such associations raise considerable funds, and may invest into research grants and drug development. The advances in LAM research in the recent years is due, for a large part, to American LAM Foundation funding. Patient associations are usually national, due to linguistics (not all patients in Europe speak English). However, some federations of patients' associations exist on an European basis. These further participate in Eurordis, a coalition of rare disease associations and national alliances of rare diseases organisations (www.eurordis.org). 


\section{Networks and reference centres}

As orphan diseases are rare, the experience of most clinicians is obviously limited. Furthermore, medical knowledge about such diseases is often scanty. Any disease first requires observational cohort studies to define its characteristics and the clinical phenotypes, thus allowing its recognition by clinicians. Pathological characterisation and biopathological studies further contribute to the individualisation of the disease. For a number of rare diseases, molecular genetic studies identify mutations more or less specific to the condition.

To gain more clinical information on an orphan disease, networks of clinician and registries collect cases and data. If many clinicians with few cases eventually contribute, large cohorts can be constituted. The clinicians participating in rare disease networks further benefit from feedback information from the studies they contributed to.

The organisation of networks depends on coordinating centres. Furthermore, such centres naturally must have extended knowledge and experience of a specific rare disease (or group of diseases). Such centres thus play a central role in the healthcare organisation for diagnosis, care and research on orphan diseases.

The French National Plan for Rare Diseases, running 2004-2008, illustrates a hitherto unique strategy for the care of orphan diseases. Ten strategic priorities have been identified, as follows: 1) to increase knowledge of the epidemiology of rare diseases; 2 ) to recognise the specificity of rare diseases; 3 ) to develop information for patients, health professionals and the general public concerning rare diseases; 4) to train professionals to better identify them; 5) to organise screening and access to diagnostic tests;
6) to improve access to treatment and the quality of healthcare provision for patients; 7) to continue efforts in favour of orphan drugs; 8) to respond to the specific needs of relatives of people suffering from rare diseases and to develop support for patients' associations; 9) to promote research and innovation on rare diseases, notably for treatment; and 10) to develop national and European partnerships in the domain of rare diseases. One cornerstone of the plan has been the designation of the reference centres for rare diseases (including, in the respiratory field, three centres for primary pulmonary hypertension, Ondine's syndrome and orphan lung diseases, respectively) (box 4). The centres apply the above strategic priorities, initiate the elaboration of guidelines and research projects, and specifically provide a source of information and help for the care of rare diseases for patients and health professionals. About 100 reference centres should be designated in the course of the plan. The concept of "official" reference centres has been accepted in five other European countries (i.e. Sweden, UK, Denmark, Belgium and Italy), using various definitions of rare diseases (two per 100,000 or lower in the UK).

A Rare Diseases Task Force working group on European centres of reference for rare diseases has been created, which aims to define and process a qualification for European centres (monthly newsletter of the rare diseases task force available at www.orphan.net/actor/EuropaNews).

\section{Cooperation of European chest physicians}

Although national actions have been developed in several EU countries to improve the care of patients with orphan lung diseases, cooperative

\section{Box 4 The GERM" O"P and Reference Centre for Orphan Lung Diseases}

The French Groupe d'Etudes et de Recherche sur les Maladies “Orphelines" Pulmonaires (GERM“0"P) was created in 1994, after French pulmonologists efficiently cooperated to collect, analyse and publish results from a series of patients with Wegener's disease [5] and primary pulmonary lymphomas [6], with 77 and 70 cases, respectively. The group currently forms a network of $>200$ chest physicians (mainly from university hospitals, but also from local hospitals and private practice physicians) and holds a registry (since 1994) with about 2,000 cases of rare orphan lung diseases. The coordinating centre in Lyon (J.F. Cordier) was designated in 2004 as a reference centre for orphan lung diseases.

Clinical studies have been published by GERM“ 0 ” $P$ in several orphan lung disease fields, including idiopathic chronic eosinophilic pneumonia [7, 8], organising pneumonia primed by radiation therapy to the breast [9], LAM [10-13], cryptogenic organising pneumonia [14], tracheobronchopathia chondrosteoplastica [15], idiopathic acute eosinophilic pneumonia [16], interstitial lung disease in amyopathic dermatomyositis, dermatomyositis and polymyositis [17]. 


\section{Box 5: European LAM actions development}

Clinical and basic research have markedly increased in the last few years (figure 4) and, by now, almost all chest physicians are aware of the existence of this very rare disease (with a prevalence of about one in 600,000 females aged $20-70$ years). They are aware, as are the radiologists, of the characteristic cystic features on chest HRCT. Many reviews and chapters in books have popularised this disorder.

Clinical studies of "large" cohorts of patients have been published in Europe, especially from UK and France, which have helped to define the clinical, imaging and prognostic features of this disease.

An ERS LAM Task Force, chaired by S. Johnson (UK) and J.F. Cordier (France), was launched at the Copenhagen ERS Congress with two objectives:

1. To define clinical guidelines for the diagnosis and care of LAM (with the participation of pathologists, surgeons and specialists in lung transplantation, specialists in tuberous sclerosis complex, etc.).

2. To establish an European network of LAM specialists in all countries, to improve diagnosis and care, and collect information from larger cohorts and prepare prospective (especially therapeutic) research and trials.

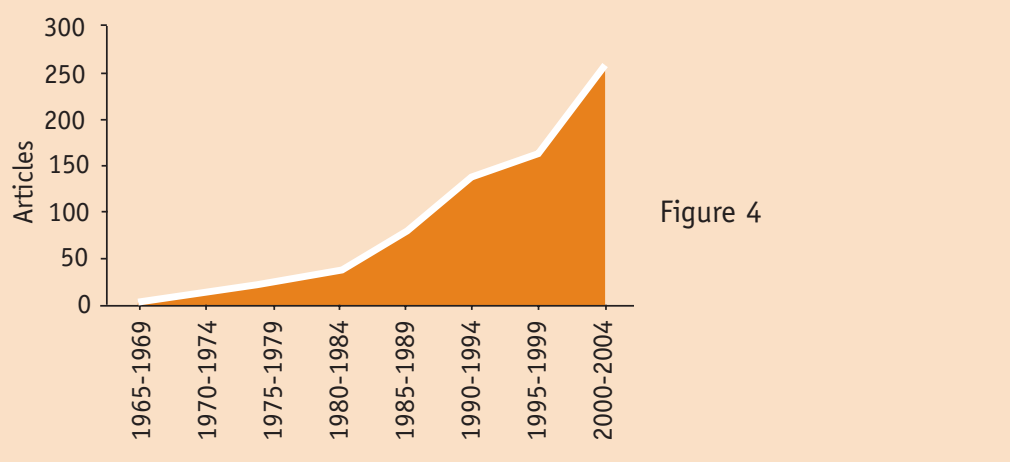

European actions are lacking. Since cohorts of patients are usually very small, no advance will be made without cooperation between chest specialists from the different European countries. Defining guidelines, establishing trans-European networks and promoting educational projects in the field are all urgently needed and these ideas are strongly supported by the patients (box 5).

\section{The future}

People affected by rare and/or orphan diseases have the same rights to access to healthcare as people who suffer from more common disorders. The development of therapeutic research and the optimisation of currently available treatments, despite financial obstacles, need specific public support throughout the world.

\section{Educational questions}

1. A rare disease in Europe affects:

a) Less than $1 / 200$ people. b) Less than $1 / 2,000$ people. c) Less than $1 / 5,000$ people.

d) Less than $1 / 50,000$ people. e) Less than $1 / 100,000$ people.

2. Which HRCT pulmonary pattern is characteristic for LAM?
a) Multiple nodules.
b) Crazy paving.
c) Multiple cysts with predominance in the upper lobes.
d) Multiple cysts throughout the lungs.
e) Honeycombing.

3. Which of the following are common in Churg-Strauss syndrome?
a) Asthma.
b) Mononeuritis multiplex.
c) Blood eosinophilia (>1.5x109 per L).
d) Vasculitic purpura of the skin.
e) Cardiomyopathy. 


\section{Suggested answers}

1.b

2.d

3. $a, b, c, d, e$

\section{References}

1. Barrett MP, Burchmore RJS, Stich A, et al. The trypanosomiases. Lancet 2003; 362: 1469-1480.

2. Couzin J. Advocating, the clinical way. Science 2005; 308: 940-942.

3. Sheridan C. EU to review rare disease drugs market exclusivity. Nature Biotechnol 2004; 22: 1061.

4. Gibson RL, Burns JL, Ramsey BW. Pathophysiology and management of pulmonary infections in cystic fibrosis. Am J Respir Crit Care Med 2003; 168: 918-951.

5. Cordier JF, Valeyre D, Guillevin L, Loire R, Brechot JM. Pulmonary Wegener's granulomatosis. A clinical and imaging study of 77 cases. Chest 1990; 97: 906-912.

6. Cordier JF, Chailleux E, Lauque D, et al. Primary pulmonary lymphomas. A clinical study of 70 cases in nonimmunocompromised patients. Chest 1993; 103: 201-208.

7. Marchand E, Reynaud-Gaubert M, Lauque D, et al. Idiopathic chronic eosinophilic pneumonia. A clinical and follow-up study of 62 cases. Medicine (Baltimore) 1998; 77: 299-312.

8. Marchand E, Etienne-Mastroianni B, Chanez $P$, et al. Idiopathic chronic eosinophilic pneumonia and asthma: how do they influence each other? Eur Respir J 2003; 22: 8-13.

9. Crestani B, Valeyre D, Roden $S$, et al. Bronchiolitis obliterans organizing pneumonia syndrome primed by radiation therapy to the breast. Am J Respir Crit Care Med 1998; 158: 1929-1935.

10. Cordier JF, Lazor R. Perspectives on lymphangioleiomyomatosis in France. In: Moss J, ed. LAM and Other Diseases Characterized by Smooth Muscle Proliferation. New York, Marcel Dekker, 1999; pp. 9-31.

11. Urban T, Lazor R, Lacronique J, et al. Pulmonary lymphangioleiomyomatosis. A study of 69 patients. Medicine (Baltimore) 1999; 78: 321-327.

12. Lazor R, Valeyre D, Lacronique J, et al. Low initial KCO predicts rapid FEV1 decline in pulmonary lymphangioleiomyomatosis. Respir Med 2004; 98: 536-541.

13. Cottin V, Vukusic S, Jouanneau E, Lazor R, Cordier JF. Should patients with lymphangioleiomyomatosis undergo screening for meningioma? Eur Respir J 2004; 24: 888-889.

14. Lazor R, Vandevenne A, Pelletier A, et al. Cryptogenic organizing pneumonia. Characteristics of relapses in a series of 48 patients. Am J Respir Crit Care Med 2000; 162: 571-577.

15. Leske V, Lazor R, Coetmeur D, Crestani B, Chatte G, Cordier JF. Tracheobronchopathia osteochondroplastica: a study of 41 patients. Medicine (Baltimore) 2001; 80: 378-390.

16. Philit F, Etienne-Mastroianni B, Parrot A, Guerin C, Robert D, Cordier JF. Idiopathic acute eosinophilic pneumonia: a study of 22 patients. Am J Respir Crit Care Med 2002; 166: 1235-1239.

17. Cottin V, Thivolet-Bejui F, Reynaud-Gaubert M, et al. Interstitial lung disease in amyotrophic dermatomyositis, dermatomyositis and polymyositis. Eur Respir J 2003; 22: 245-250. 